\title{
Pratiques
}

Linguistique, littérature, didactique

141-142| 2009

La synonymie

\section{La quasi-synonymie du passé composé et du passé surcomposé dit « régional »}

Denis Apothéloz

\section{(2) OpenEdition}

\section{Journals}

Édition électronique

URL : https://journals.openedition.org/pratiques/1308

DOI : 10.4000/pratiques.1308

ISSN : 2425-2042

Éditeur

Centre de recherche sur les médiations (CREM)

\section{Édition imprimée}

Date de publication : 15 juin 2009

Pagination : 98-120

\section{Référence électronique}

Denis Apothéloz, «La quasi-synonymie du passé composé et du passé surcomposé dit « régional » », Pratiques [En ligne], 141-142 | 2009, mis en ligne le 20 juin 2014, consulté le 10 mars 2023. URL :

http://journals.openedition.org/pratiques/1308; DOl : https://doi.org/10.4000/pratiques.1308 


\title{
La quasi-synonymie du passé composé et du passé surcomposé dit « régional »
}

\section{Denis Apothéloz}

\author{
Université de Nancy 2 et ATILF (UMR 7118)
}

Au regard du thème de la présente livraison de Pratiques, l'objet de cet article paraîtra quelque peu particulier. Nous y abordons un fait de synonymie touchant non pas le lexique mais la grammaire, plus exactement le système des temps verbaux du français. Les lignes qui suivent sont en effet consacrées à certains emplois du passé composé et du passé surcomposé. L'un des termes de la synonymie examinée ici (le passé surcomposé) a, de surcroît, la réputation d'être une forme plutôt rare et circonscrite à certaines zones géographiques. Tout cela pourrait conférer au problème étudié une dimension plutôt marginale, du moins relativement au thème du présent numéro. Nous tenterons cependant de montrer que le type de synonymie étudié dans cet article pose des problèmes théoriques et méthodologiques particuliers.

Cette étude est organisée comme suit. Dans une première partie, nous présentons la problématique des énoncés existentiels, qui constitue la toile de fond de l'ensemble de nos analyses. Nous résumons ensuite, dans une deuxième partie, l'état des connaissances sur les formes surcomposées. Après avoir isolé une valeur particulière, que nous appelons énoncé d'expérience, nous analysons dans une troisième partie les principales propriétés sémantiques et discursives de ces énoncés. Sur cette base nous menons, dans la quatrième partie, une exploration sémantique des points communs et des différences entre le surcomposé dit « régional » et le passé composé employé en énoncé d'expérience.

Comme le font beaucoup d'aspectologues, nous utiliserons le terme de « situation » pour désigner le procès dénoté par la forme verbale ou prédicative étudiée, quelle que soit la nature de ce procès.

Pour des raisons méthodologiques évidentes, nous avons conservé l'orthographe d'origine dans tous les exemples (de même que dans le titre de l'article de Clédat!). Cet avertissement vaut surtout pour les exemples issus de forums sur Internet et pour ceux trouvés dans un ouvrage de géographie datant de la fin du $\mathrm{XVIII}^{\mathrm{e}} \mathrm{s}$. Les exemples non référencés ont été forgés pour les besoins de la démonstration ou résultent de manipulations d'exemples référencés. 


\section{Les énoncés existentiels-événementiels}

La notion d'énoncé existentiel, telle qu'elle a été définie par Jespersen (1924), renvoie à une grande variété de types d'énoncés, qu'il est impossible de présenter dans le cadre de ce travail. Ceux qui retiendront notre attention, et à partir desquels nous aborderons notre problème de synonymie, sont parfois caractérisés comme énoncés « existentiels-événementiels » (Lambrecht, 1994, Van de Velde, 2006). On entend par là un énoncé dont la fonction communicationnelle principale est de prédiquer qu'une situation a (a eu) lieu, ou, comme disent les logiciens, « est (a été) le cas ». Commençons par examiner un exemple commenté par Kleiber (2001 : 49) dans un long article sur les indéfinis :

(1) Des inconnus ont cambriolé la maison de Léa.

Il s'agit là d'un exemple caractéristique d'énoncé existentiel-événementiel. On peut en gloser la signification ainsi : "ily a des inconnus qui ont cambriolé la maison de Léa ", ou même " il y a que des inconnus ont cambriolé la maison de Léa ". Dans ces gloses, le tour il y a (que) expose, i.e. manifeste par une forme linguistique, la valeur d'existence et indique son statut de prédication principale (quoique non explicitement exprimé). Dans cet exemple, le SN indéfini des inconnus est lui-même en emploi existentiel (par opposition aux emplois dits " partitifs » des indéfinis ${ }^{(1)}$ ), et participe de l'interprétation qui vient d'être donnée. Cet énoncé répond typiquement à des questions comme Que se passe-t-il ?, Pourquoi toute cette agitation?, etc.

Cependant le type d'énoncé existentiel que nous étudierons ici est un peu différent. Soit (2), version légèrement transformée de (1) :

(2) La maison de Léa a été cambriolée par des inconnus.

Cet exemple admet deux lectures au moins. La première correspond à la formulation $(2 \mathrm{a})$, la seconde à $(2 \mathrm{~b})$ :

(2a) Il y a la maison de Léa qui a été cambriolée par des inconnus.

(2b) Il est (déjà) arrivé que la maison de Léa soit cambriolée par des inconnus.

Dans la lecture (2b), il s'agit bien toujours d'une valeur existentielle-événementielle (on asserte d'une situation qu'elle a eu lieu), mais la localisation temporelle est ici différente : la prédication d'existence porte non pas sur une situation unique et temporellement localisée, mais sur une possible série de situations identiques, cette série étant localisée dans le passé et comportant au moins une occurrence de la situation. La localisation temporelle de cette possible série est donc un intervalle ouvert, qu'on pourrait d'ailleurs mettre en évidence, dans (2b), au moyen d'un complément comme dans le passé : Il est (déjà) arrivé dans le passé que la maison de Léa soit cambriolée par des inconnus. En langue orale, l'énonciation de (2) avec ce type de signification est en principe soulignée par un contour prosodique particulier, consistant en une emphase sur l'auxiliaire. Leech (1971: §56) avait déjà signalé ce fait sur l'anglais. Notons encore que l'adverbe déjà de (2b) doit être distingué de déjà indiquant une « survenance précoce », comme dans

(1) Celui qu'on a par exemple dans Des députés ont voté blanc (Kleiber 2001 : 61), où des députés est en emploi partitif-i.e. opérant une partition entre les députés qui ont voté blanc et les autres, cf. la glose sémantique "parmi le députés, certains ont voté blanc». 
il est déjà trois heures (Mosegaard Hansen, 2000). Le premier est proprement existentiel et est nié par jamais (cf.la maison de Léa n'a jamais été cambriolée par des inconnus); le second n'a rien à voir avec la notion d'existence et est nié par pas encore (cf. il n'est pas encore trois heures). Selon Mosegaard Hansen, déjà existentiel n'apparaît qu'avec les temps composés ${ }^{(2)}$. Nous reviendrons plus loin sur le rôle des adverbiaux dans la détermination de cette seconde lecture existentielle.

On s'aperçoit, avec (2) et les interprétations qui viennent d'être décrites, que la lecture existentielle n'est nullement dépendante du caractère indéfini du SN sujet. En fait, cette interprétation est tributaire du temps verbal (ici le passé composé). Dans les travaux aspectologiques portant sur l'anglais, langue dans laquelle le present perfect a des emplois analogues, l'interprétation correspondant à (2b) est appelée « parfait indéfini » (Leech, 1971), « parfait existentiel» (McCawley, 1971) ou encore «parfait d'expérience» (Comrie, 1976). S'agissant du français, Vet (1992) utilise quant à lui l'expression de «passé composé expérientiel». Compte tenu du fait que, comme nous le verrons, le passé composé n'est qu'un élément parmi d'autres dans la construction de cette signification, nous préférerons quant à nous parler d' " énoncés d'expérience » pour désigner le type d'énoncé existentiel-événementiel correspondant à la lecture (2b) de (2). Dans la terminologie utilisée ici, les énoncés d'expérience sont donc une variété d'énoncés existentiels ${ }^{(3)}$.

Nous avons montré ailleurs que la valeur mise en évidence dans (2b) pourrait, dans certaines régions de la francophonie, être exprimée également par une forme surcomposée (Apothéloz, à par.). Cela donnerait (3) :

(3) La maison de Léa a eu été cambriolée par des inconnus.

Avant d'analyser ce type de formulation, il convient toutefois de rappeler quelques informations générales sur le passé surcomposé.

\section{Le passé surcomposé}

Sans être extrêmement nombreux, les travaux consacrés aux formes verbales surcomposées, et notamment au passé surcomposé, n'en sont pas rares pour autant. Il faut dire que la description du passé surcomposé pose des problèmes particulièrement complexes, dus au fait que ce tiroir présente des phénomènes de variation à plusieurs niveaux : morphologique, sémantique, géographique et sociolinguistique. Dans les grandes lignes, la situation est la suivante :

(a) Bien qu'il soit, en tant que forme, généralement compris sur tout le terri-

(2) Cet auteur appelle « itératif » cette valeur de déjà, mais cette appellation peut prêter à confusion, dans la mesure où l'itérativité n'est ici que potentielle. Nous y reviendrons.

(3) Les énoncés d'expérience ont, en anglais, une plus grande visibilité grammaticale. Dans cette langue, en effet, le verbe utilisé pour traduire un énoncé comme Bill a été en Amérique n'est pas le même, selon que le present perfect est interprété comme un présent résultatif (« accompli ») ou comme un parfait d'expérience (Comrie, 1976). La formulation Bill has gone to America exprime un présent résultatif et implique donc, soit que "Bill est actuellement en Amérique ", soit que "Bill est en train de se rendre en Amérique ». Tandis que la formulation Bill has been to American'a pas ces implications et pourrait être traduite en français par : Il est déjà arrivé à Bill d'aller en Amérique. En anglais, tout se passe donc comme si, pour le signifié « aller », la valeur existentielle était grammaticalisée. Ce fait explique peut-être pourquoi les travaux sur le parfait existentiel sont beaucoup plus nombreux dans la tradition aspectologique anglo-saxonne que dans la tradition française. 
toire de la francophonie, le surcomposé n'est pas pratiqué sur tout ce territoire ; il s'agit donc d'une forme qui, dans les pratiques linguistiques, est en quelque sorte traversée par la distinction entre compétence active et compétence passive.

(b) Il présente une panoplie d'emplois, de « valeurs », qui n'est pas la même sur tout le territoire francophone. Certains emplois sont confinés à certaines aires géographiques. C'est justement le cas de la variante qui nous intéresse ici, qui est limitée au Midi de la France et à l'aire franco-provençale, Suisse romande comprise.

(c) Cette répartition géographique s'accompagne de divers phénomènes sociolinguistiques (stigmatisation) sur la description desquels les linguistes sont d'ailleurs loin de s'accorder. Par exemple, s'agissant de la variante qui nous intéresse, elle est aujourd'hui pratiquement absente de l'écrit « normé », mais réapparaît depuis une quinzaine d'années par le canal d'Internet dans les écrits électroniques (forums, blogs, etc.).

Compte tenu de l'objectif de cet article, seule la dimension sémantique, aspectuo-temporelle, va nous retenir ici. Les autres dimensions ne seront évoquées que lorsque cela sera indispensable. Pour le reste, le lecteur pourra se référer aux travaux suivants : Foulet (1925), Clédat (1926), Tesnière (1939), Cornu (1953), Stefanini (1954), Dauzat (1954), Walter (1981), Hill (1984), Jolivet (1984, 1986), Ayres-Bennett et Carruthers (1992), Carruthers (1994, 1998, 1999), Engel (1996), Wilmet (1997), Sthioul (2006), Schaden (2007). De même, nous ne parlerons pas des autres tiroirs surcomposés : plus-que-parfait surcomposé, futur antérieur surcomposé, conditionnel surcomposé, etc.

La très grande majorité des descriptions qui ont été données du surcomposé s'accordent pour distinguer deux emplois principaux. Ces emplois sont, de façon tout à fait caractéristique, associés à des contextes grammaticaux spécifiques.

Le premier est celui qu' on observe, d'une part dans les subordonnées temporelles en quand, lorsque, dès que, aussitôt que, une fois que, après que, etc., d'autre part dans des propositions dans lesquelles la forme verbale est modifiée par un adverbial comme vite, rapidement, bientôt, ou un syntagme prépositionnel ayant un effet de « précipitation » de 1'action (Barceló et Bres, 2006). Cet emploi est en général considéré comme standard, non spécifique à une région particulière. En voici quelques exemples caractéristiques.

(4) [...] quand elle a eu trouvé son équilibre, elle a dit, avec sa voix blanche de ces moments-là : - Oncle Thian vous fait dire qu'il est bien arrivé. (D. Pennac, Lapetite marchande de prose, Frantext)

(5) Dès qu'on a eu échangé quelques phrases, je me suis sentie aussitôt en complicité. (V. Therame, Bastienne, Frantext)

(6) - Il est arrivé ici presque aussitôt que vous avez eu quitté la tour, Monsieur Sainclair. (G. Leroux, Le parfum de la dame en noir, Frantext)

(7) Le vent de la réalité a eu vite fait de balayer ces illusions, et ce fut la fin du premier épisode de la conférence de Genève. (P. Mendès-France, Euvres complètes, Frantext)

(8) En cinq minutes, il a eu disparu. (H. Alain-Fournier, Correspondance avec J. Rivière, Frantext)

Dans ce type de contexte, le surcomposé désigne presque toujours l'état consécutif au procès dénoté par le verbe. Sur le plan aspectuo-temporel, il a donc une va- 
leur de passé résultatif. Dans (4), la forme verbale désigne la portion temporelle qui débute à partir du moment où la personne désignée a trouvé son équilibre; dans (5), celle à partir de laquelle un certain nombre de phrases ont été échangées; dans (6), celle à partir de laquelle la personne désignée par vous n'est plus dans la tour. Dans ces trois exemples, le surcomposé est lié à l'expression d'une corrélation temporelle entre deux situations. Il en va de même, mais de façon implicite, dans les deux exemples suivants, où on retrouve la même valeur aspectuo-temporelle : dans (7), la forme verbale désigne la temporalité qui débute à partir du moment où les illusions sont balayées ; et dans (8), celle à partir de laquelle l'individu désigné par il n'est plus visible (cet exemple doit être compris comme signifiant non pas " il a mis cinq minutes pour disparaître », mais plutôt " au bout de cinq minutes il était disparu »). Avec des adverbes comme vite et rapidement, on observe en outre que le verbe lexical est parfois auxilié par la proforme faire, comme a eu vite fait de balayer dans (7).

Ces environnements grammaticaux font évidemment penser au passé antérieur. Et en effet, il a été noté depuis longtemps que dans cet emploi, le passé surcomposé joue relativement au passé composé le même rôle que le passé antérieur relativement au passé simple ${ }^{(4)}$. A cet égard, on peut dire que c'est une sorte de «passé antérieur de discours ».

Le second emploi n'a plus rien à voir avec la corrélation de deux situations. Ici, d'ailleurs, la substitution avec le passé antérieur n'est plus possible (ou alors le sens produit serait totalement différent). C'est précisément ce surcomposé qui n'est pratiquement utilisé que dans le Midi de la France et dans la région francoprovençale, Suisse romande comprise, où sa vitalité semble assez importante ( $c f$. Bürgi, 2007). L'exemple (3) ci-dessus, issu d'une manipulation, était de ce type. En voici d'autres :

(9) Tous les Habitans sont Catholiques Romains. L'Evêque, souverain du Pays, a des revenus très-considérables. Il a eu mis dix mille hommes sur pied, en tems de guerre. (H. Descombes, Géographie universelle, T.1, Lausanne, 1790, p. 433)

(10) Les chaleurs de l'Eté n'y sont pas moins grandes que le froid de l'hyver; [...] Elles n'y sont, d'ailleurs, tempérées par aucun vent. On assure qu'elles ont eu mis des forêts en feu; \& que ces incendies ont, quelquefois, duré plusieurs mois, \&, même, plusieurs années, sans qu'on ait pû les éteindre. (ibid, p. 169)

(11) Ça serait un peu correct de ne pas mettre tout ce qui constitue l'alimentation bio dans le même panier. J'ai eu trouvé, dans un magasin bio, des steaks hachés sous barquette indiquant $12 \%$ de bio. Le reste est constitué de quoi ? (http ://www.tarot-numerologie.fr/)

(12) Enfin, en ce qui concerne mes vacances, $j$ 'en ai eu passé de meilleures, n'étant pas parti cette année. (http ://forums.france3.fr/france3/france3jt/)

Ce surcomposé a reçu diverses appellations, dans lesquelles les auteurs font généralement référence aux particularités géographiques de cette forme. Dauzat (1954) le qualifiait de surcomposé « provençal » (qu'il opposait au surcomposé « français »), et Foulet (1925), de surcomposé « régional » (opposé à « général »). Cette dernière appellation est régulièrement utilisée aujourd'hui encore. Quant à Cornu (1953), il le désignait tantôt par l'expression de surcomposé « absolu », tan-

(4) Par les grammairiens au moins dès le XVIII ${ }^{\mathrm{e}}$ siècle (par ex. Beauzée), mais aussi par Clédat (1926), Frei (1929), Gougenheim (1939), Tesnière (1939), Cornu (1953), Benveniste (1959), Martinet (1979), Weinrich (1989), pour n'en citer que quelques-uns. 
tôt «à valeur spéciale». «Absolu» renvoie au fait que, contrairement aux emplois du premier contexte, ce surcomposé n'est pas lié à l'expression d'une corrélation temporelle de deux situations ${ }^{(5)}$.

Comme on le voit, ces appellations ne parviennent pas à cacher une certaine impuissance à décrire de façon satisfaisante les propriétés sémantiques, éventuellement pragmatiques, de ces formes. Parmi les stéréotypes les plus répandus et les plus récurrents circulant sur la sémantique du passé surcomposé, on peut relever l'idée qu'il exprime un passé lointain ou révolu, une certaine subjectivité voire une certaine affectivité relativement à la situation désignée, ou encore qu'il met en évidence la nature exceptionnelle de cette situation. Il s'est quand même trouvé certains auteurs pour mettre en doute le bien-fondé de certaines de ces descriptions, par exemple celle consistant à attribuer au surcomposé la vertu d'exprimer une certaine subjectivité (e.g. Sthioul 2006).

Cela dit, il ne fait aucun doute que la valeur des surcomposés de (9)-(12) est très différente de celle des exemples (4)-(8) du premier contexte. Il n'y a dans (9)-(12) aucune résultativité dans le passé. De fait, ces passés surcomposés sont des parfaits existentiels et les énoncés qu'ils forment sont typiquement des énoncés d'expérience, au sens défini plus haut. Les gloses au moyen du tour impersonnel il est arrivé que/de, qui explicitent fidèlement le sens de ces énoncés, le font voir clairement:

(9a) Il est arrivé qu'il mette dix mille hommes sur pied, en tems de guerre.

(10a) On assure qu'il est arrivé qu'elles mettent des forêts en feu; \& que ces incendies ont, quelquefois, duré plusieurs mois...

(11a) Il m'est arrivé de trouver, dans un magasin bio, des steaks hachés sous barquette indiquant $12 \%$ de bio.

(12a) Enfin, en ce qui concerne mes vacances, il m'est arrivé d'en passer de meilleures, n'étant pas parti cette année.

Les exemples (9)-(12) n'ont pas été choisis au hasard. Les deux premiers font voir que le statut sociolinguistique de cette forme a vraisemblablement évolué et que, dans cet emploi, le surcomposé n'a pas toujours fait l'objet d'une stigmatisation normative ${ }^{(6)}$. De nos jours, dans des écrits en principe aussi normés qu'un traité de géographie, un surcomposé de ce type serait assez peu probable. Il n'en demeure pas moins que les formulations de (9) et (10) pourraient être produites aujourd'hui encore dans un registre moins « surveillé ».

Nous voici donc au cœur de notre problème : un fait de synonymie, ou de quasisynonymie, entre deux tiroirs verbaux : le passé composé dans les énoncés d'expérience, et le passé surcomposé dans le même type d'emploi. La question qui se pose maintenant est de savoir s'il s'agit d'une synonymie vraie et, si la réponse est négative, de mettre au jour ce qui différencie ces deux formes.

Pour pouvoir répondre en toute connaissance de cause à cette question, il est nécessaire d'examiner plus en détail le fonctionnement des énoncés d'expérience.

(5) Les appellations qui font référence à la diatopie ont certes l'avantage d'une certaine objectivité, mais à condition d'être fondées sur des données correctes. De ce point de vue, l'appellation proposée par Dauzat est tout simplement erronée. De plus, en opposant «provençal» à « français », cet auteur assimilait manifestement le «centre » à la norme, et la «périphérie » au non-respect de celle-ci, ce qui parait évidemment des plus problématiques.

(6) Ce type de surcomposé est attesté depuis le XIV es. au moins (cf. Cornu, 1953 ; NilssonEhle, 1953). 
Tel est 1'objectif de la section suivante. Nous appellerons désormais « surcomposé-E » le passé surcomposé des exemples (9)-(12) ${ }^{(7)}$.

\section{Passé composé en énoncé d'expérience et surcomposé-E}

Nous allons maintenant tenter d'aller un peu plus loin dans notre exploration des énoncés d'expérience. Notre investigation consistera principalement à poser la question suivante : quelles sont les propriétés sémantiques, pragmatiques voire discursives, de ces énoncés?

\subsection{Collocation avec certains adverbiaux}

Tout d'abord, on observe que l'interprétation d'un énoncé au passé composé comme énoncé d'expérience est fréquemment associée à la présence de certains adverbiaux ${ }^{\left({ }^{8}\right)}$. Il s'agit principalement de quantifieurs de fréquence (déjà à valeur existentielle, unjour, une fois, toujours, souvent, parfois, rarement, pas une seule fois, jamais, etc.) ou d'expressions localisant le procès dans un passé plus ou moins lointain et indéterminé (autrefois, jadis, il y a longtemps, etc.). Cette corrélation a été notée par de nombreux auteurs, pour le français comme pour l'anglais - e.g. Leech (1971), Comrie (1976), Dahl (1985), Guentchéva (1990), Desclés \& Guentchéva (2003), Karolak (2007). Leech observe même qu'en anglais, l'interprétation existentielle d'un énoncé au present perfect est rare sans le concours d'un adverbe comme ever, never ou before, qui « renforcent » (telle est l'expression utilisée par cet auteur) cette interprétation. En voici quelques illustrations :

(13) J'ai déjà eu un enfant, et je sais que ce moment est terrible où, tête baissée, il creusera, enfoncera mon utérus, jusqu'au moment où il sera assez large pour y passer tout entier. (M. Duras, Cahiers de la guerre, 301)

(14) Mes travaux de presse consistant à traiter des sujets sur dossiers, j'ai rarement voyagé pour aller chercher le scoop dans les bas-fonds de cinéma. (J.-L. Degaudenzi, Zone, Frantext)

(15) Elles trottinent sur leurs courtes jambes en balançant les corolles de leurs jupettes que rien ne distingue - sinon la taille - des vêtements des femmes adultes. C'est vrai aussi de leur comportement. J'ai souvent vu des fillettes très jeunes - trois ou quatre ans - avoir à l'égard des hommes une attitude très typiquement et comiquement féminine, sans équivalent dans la conduite des petits garçons à l'égard des femmes. (M. Tournier, Le roi des Aulnes, Frantext)

(16) J'ai parfois surpris, chez cet homme vraiment supérieur, un indéfinissable ton de mépris en face de Robert. (A. Gide, Robert, Frantext)

(17) As-tu jamais vu un petit poisson qui essaie de remonter un courant rapide, et que la force du courant maintient à la même place, immobile et frémissant. (H. de Montherlant, Pasiphaé, Frantext)

On trouve aussi ces adverbiaux avec le surcomposé-E, quoique cette association paraisse moins systématique :

(7) A notre connaissance, la seule étude qui établisse un rapprochement entre les surcomposés et les énoncés existentiels est l'ouvrage de Squartini sur les périphrases verbales. Cet auteur, commentant des exemples de surcomposés, explique qu'il y a dans certains d'entre eux "rather an "experiential" flavour " $(1998: 205)$.

(8) «Fréquemment » est une estimation purement intuitive, car nous ne disposons pas de données quantitatives sur cette collocation. Les raisons en sont données plus loin. 
(18) - Tu devrais faire une cure!

- Tu crois!

- Tu l'as déjà eu fait, ça t'a pas trop mal réussi ! (ex. J.-P. Goudaillier, in Walter $1981: 43)$

(19) Il est vrai que certaines fois j'ai eu regretté de m'être dévoilé ainsi devant un(e) inconnue (http ://forums.voissa.com/)

(20) J'ai eu trouvé parfois des transistors mal montés contre ce qui leur sert de refroidisseur, d'ou je suppose une mauvaise dissipation thermique. Il faut les monter avec de la graisse thermique, les fixer et les souder à la fin. (http ://www.abcelectronique.com/)

Il y a toutefois ici une différence entre les deux tiroirs. Alors qu'avec le passé composé, la présence de l'adverbial peut s'avérer indispensable pour assurer l'interprétation de l'énoncé comme énoncé d'expérience, avec le surcomposé-E il en va différemment. L'extrait (16), par exemple, tel qu'il a été isolé de son environnement, devient ambigu si on retire l'adverbe parfois : le passé composé peut alors y être interprété soit comme un prétérit (i.e. désignant une situation particulière et temporellement définie) soit comme un parfait d'expérience. L'exemple (14) sans son adverbe n'est pas très clair non plus à l'égard de cette distinction. En revanche, on peut sans difficulté supprimer déjà dans (18), certaines fois dans (19), et parfois dans (20), sans perte de la valeur d'énoncé d'expérience. Les locuteurs pour lesquels ce type de surcomposé est familier considéreront même que ces adverbiaux sont pratiquement redondants dans ces trois exemples !

Il semble donc qu'il y ait là une différence de fonctionnement entre nos deux tiroirs. C'est un point important, sur lequel on reviendra.

\section{2. «Une fois au moins dans le passé »}

Les énoncés d'expérience signalent, à défaut d'information complémentaire, que la situation désignée a eu lieu une fois au moins dans le passé (Comrie 1976, Dahl 1985, Guentchéva 1990, Karolak 2007). Il s'agit bien d'une valeur «par défaut » puisque, comme on vient de le voir, un adverbial quantifieur de fréquence (rarement, plus d'une fois, etc.) peut venir la spécifier. Elle implique cependant une sorte d'itérativité potentielle.

Cette « répétabilité » qu'impliquent les énoncés d'expérience est une propriété importante. Elle revient, de fait, à présenter la situation comme ressortissant à un type. Comme l'écrit Dahl (1985:141), "the sentence must concern a generic activity, state, etc., rather than an individual, or specific one ». Cette caractéristique sémantique a pour conséquence le fait suivant: une situation qui n'est pas concevable comme répétable ne peut pas être désignée au moyen d'un énoncé d'expérience. Contrairement à Je suis allé au Yémen, un énoncé comme Je suis né au Yémen n'a pas d'interprétation comme énoncé d'expérience.

Cette propriété sémantique caractérise aussi bien le passé composé en énoncé d'expérience que le surcomposé-E. Elle est au fondement des énoncés d'expérience tels que nous les avons définis. Il découle de cette valeur par défaut deux propriétés étroitement solidaires, présentées sous 3.3. et 3.4.

\subsection{Absence de localisation précise dans le passé}

Les énoncés d'expérience désignent une situation qui n'est pas localisée précisément dans le temps. La seule chose qu'on sache à son sujet est qu'elle s'est pro- 
duite dans le passé. C'est la raison pour laquelle cet emploi a parfois été désigné comme un parfait indéfini (Leech 1971, à propos du present perfect anglais en emploi existentiel). Il en résulte que ces énoncés sont incompatibles avec une expression indiquant une localisation temporelle précise. Toute expression de localisation y sera automatiquement interprétée comme indiquant un intervalle temporel incluant la situation mais ne la localisant pas au sens strict du terme.

Ici encore, il ne nous semble pas y avoir de différence entre le passé composé en énoncé existentiel et le surcomposé-E. Ils expriment le même type d'indéfinitude.

\subsection{Intervalle de validation}

Les énoncés d'expérience sont nécessairement associés à un intervalle temporel, celui-là même à l'intérieur duquel la situation est dite s'être produite. On l'appellera intervalle « de validation », car c'est à l'intérieur de cet intervalle seulement que la prédication d'existence est valide ${ }^{(9)}$.

Il s'agit, à défaut d'information complémentaire, d'un intervalle ouvert, que limite à droite le présent de la parole. Très souvent cet intervalle est implicite et doit être construit à partir de divers indices contextuels. Il peut aussi être spécifié. Par exemple, sa borne initiale aussi bien que sa borne terminale peuvent être précisées. Ainsi, l'extrait (16) ci-dessus pourrait avoir la forme (16a) ou (16b) sans pour autant perdre sa valeur d'énoncé d'expérience : dans (16a), c'est la borne initiale qui est spécifiée ; dans (16b), c'est la borne terminale.

(16a) Depuis cet incident, j'ai parfois surpris, chez cet homme vraiment supérieur, un indéfinissable ton de mépris en face de Robert.

(16b) Avant que ne surviennent ces tragiques événements, j'ai parfois surpris, chez cet homme vraiment supérieur, un indéfinissable ton de mépris en face de Robert.

Quand aucune information complémentaire n'est donnée concernant cet intervalle, et que le référent du sujet grammatical du verbe est un individu humain, 1'intervalle correspond assez souvent à une information générale qu' on pourrait paraphraser par 1'expression : au cours de mon (ton, son, etc.) existence.

La présence de cet intervalle est commune au passé composé en énoncé d'expérience et au surcomposé-E. Néanmoins, compte tenu de certains emplois qui sont faits du surcomposé-E, dans lesquels ce qui est dit du passé est mis en contraste avec le présent, on est conduit à se demander si l'intervalle de validation est prédéfini de la même manière par ces deux tiroirs. On y reviendra dans la section suivante.

\subsection{Glose il est arrivé que/de...}

Nous avons vu qu'il est possible d'expliciter la valeur particulière des énoncés d'expérience en les paraphrasant par enchâssement dans le tour impersonnel il est arrivé que/de. Voyons-le sur les exemples de passés composés donnés plus haut, repris ci-dessous de façon raccourcie.

(13) J'ai déjà eu un enfant, et je sais que ce moment...

(13a) Il m'est déjà arrivé d'avoir un enfant, et je sais que ce moment...

(14) j'ai rarement voyagé pour aller chercher le scoop...

(14a) il m'est rarement arrivé de voyager pour aller chercher le scoop...

(9) Vet (1992: 49) appelle cet intervalle « domaine de quantification». 
(15) j'ai souvent vu des fillettes très jeunes [...] avoir à l'égard des hommes une attitude...

(15a) il m'est souvent arrivé de voir des fillettes très jeunes [...] avoir à l'égard des hommes une attitude...

(16) J'ai parfois surpris, chez cet homme vraiment supérieur, un indéfinissable ton de mépris...

(16a) Il m'est parfois arrivé de surprendre, chez cet homme vraiment supérieur, un indéfinissable ton de mépris...

(17) As-tu jamais vu un petit poisson qui essaie de remonter un courant rapide...

(17a) Est-ce qu'il t'est jamais arrivé de voir un petit poisson qui essaie de remonter un courant rapide...

Le lecteur pourra vérifier que cette même paraphrase permet de restituer la signification des surcomposés-E des exemples (18)-(20). Le tour impersonnel est assez souvent rapporté explicitement au sujet «expérimenteur » : il m'est arrivé de..., il lui est arrivé de..., il nous est arrivé de..., etc.

Le fait que le tour il est arrivé que/de produise une paraphrase fidèle du sens de l'énoncé original est une observation importante, faite d'ailleurs depuis longtemps déjà ( $c f$. par exemple Foulet, 1925 ; Frei, 1929). Elle montre que les énoncés d'expérience comportent sémantiquement cette prédication. Dans les paraphrases (13a)-(17a), l'expression il est arrivé que/de ne fait au fond qu'exhiber la prédication existentielle qui est implicite dans (13)-(17).

\subsection{Résultativité pragmatique}

Les énoncés d'expérience présentent une genre particulier de résultativité. Pour le faire voir, commençons par examiner cette notion.

Observons tout d'abord que tous les verbes ne sont pas également prédisposés à produire de la résultativité. Ainsi, il est frappant de constater que des verbes comme sortir ou s'endormir se prêtent particulièrement bien à mettre en évidence un état résultant lorsqu'ils sont fléchis à un tiroir composé, ce qui n'est pas le cas de verbes comme courir ou heurter. Les énoncés il est sorti, ou il s'est endormi, sont régulièrement produits comme des présents résultatifs pour signifier respectivement les états « être sorti » et « être endormi »; mais il en va autrement des énoncés il a couru ou il a heurté le trottoir. Quand de tels énoncés sont produits comme des présents résultatifs, l'état résultant n'a pas la même nécessité sémantique ni la même prévisibilité qu'avec des verbes comme sortir ou s'endormir. On peut certes dire, en visant un état résultant, il a couru; mais l'état visé sera alors toujours lié à des facteurs contingents et ne découlera pas du sens du verbe. Il correspondra à des informations dépendantes du contexte, et donc variables, comme : "il est essoufflé ", "il est en sueur », "il est arrivé à l'heure », etc. L'énoncé il a courupeut être produit pour attirer l'attention sur des états de ce type, et par exemple pour en donner une explication. Le même type de raisonnement pourrait être fait pour l'exemple il a heurté le trottoir.

En d'autres termes, alors que l'information «il dort » peut être inférée directement (et sans risque d'erreur) de l'énoncé il s'est endormi employé pour signifier un constat dans le présent, il n'en va pas de même de l'information " il est essoufflé » relativement à l'énoncé il a couru: "être endormi » est une composante du 
sens même du verbe s'endormir, ce qui n'est pas le cas de "être essoufflé » relativement au verbe courir.

Le paramètre sémantique - plus précisément aspectuel-qui est ici concerné est la transitionnalité au sens de Vet (1992) : sortir et $s$ 'endormir ont une signification typiquement transitionnelle (ils désignent un changement d'état) et définissent par conséquent leur propre état résultant; ce qui n'est pas le cas de courir ou heurter, dont le sens ne comporte aucune idée de transitionnalité.

En reprenant une distinctions que nous avons faite ailleurs (Apothéloz et Nowakowska, 2009), nous appellerons résultativité « sémantique » le type de relation qu'il y a entre il s'est endormi et " il dort » (le contenu de l'état résultant découle alors du sens même du verbe) ; et résultativité « pragmatique » le type de relation qu'il y a entre il a couru et "il est essoufflé », il a couru et "il est arrivé à l'heure ", etc. (le contenu de l'état résultant doit ici être construit par inférence).

Il est intéressant de noter que ces deux types de résultativités ne sont pas exclusifs. L'énoncé il s'est endormi peut, outre le fait de désigner l'état résultant sémantiquement impliqué par le verbe (i.e. "il dort»), vouloir désigner une autre conséquence, elle pragmatique au sens ci-dessus, de cet état : par exemple "il est complètement immobile ", "s sa respiration est régulière ", et d'autres conséquences de ce type.

Revenons aux énoncés d'expérience et au passé composé. On décrit souvent les divers emplois du passé composé à partir de deux valeurs de base (e.g. Waugh, 1987 ; Desclés \& Guentchéva, 2003 ; voir aussi la revue de Ritz, 2002) : l’une qui est processuelle et entièrement tournée vers le passé (dite « inaccomplie » dans la tradition grammaticale française), l'autre qui est résultative et tournée vers le présent (dite « accomplie ») ${ }^{(10)}$. Il est intéressant d'observer que les énoncés d'expérience résistent à cette distinction. Plus exactement, ils pointent simultanément, et avec une intensité égale, en direction du passé et en direction du présent : en direction du passé, parce que l'intervalle de validation à l'intérieur duquel ils prédiquent l'existence d'une situation est localisé dans le passé ; et en direction du présent, parce que ces énoncés visent in fine à attirer l'attention sur des conséquences actuelles de cette situation, observées ou observables au moment de la parole. C'est d'ailleurs précisément ces conséquences que vise à saisir 1'appellation de parfait d'expérience. Dans plusieurs exemples donnés plus haut, cette dimension d'expérience est tout à fait évidente.

En fait, les énoncés d'expérience au passé composé et au surcomposé-E visent à asserter l'existence d'une situation passée, mais seulement dans la mesure où cette existence est pertinente dans le présent sous forme de traces psychologiques, d'expérience, etc. Ces deux tiroirs actualisent donc ici une variante de la valeur aspectuo-temporelle de «présent résultatif» ${ }^{(11)}$.

Ces explications conduisent au constat que les énoncés d'expérience échappent à la distinction aspectuelle « processuel » VS « résultatif», étant à la fois processuels et résultatifs ${ }^{(12)}$. Cependant leur résultativité est d'un type particulier. Elle

(10) Voir cependant Vet (1992), qui distingue trois types majeurs de passés composés. Le vocabulaire que nous utilisons ici (processuel / résultatif) est emprunté à Confais (1995).

(11) C'est ce type de résultativité, nous semble-t-il, que Sthioul (2006:125) appelle « un état résultant de second niveau ", s'agissant du surcomposé.

(12) Pour cette raison, il est très difficile d'en rendre compte au moyen d'un modèle comme celui des trois « points » (S, R et E) de Reichenbach (1947), ou de modèles dérivés (par exemple Gosselin, 2005). 
n'est pas issue des propriétés aspectuo-temporelles du lexème verbal : elle n'est donc pas sémantique, mais pragmatique, calculée en fonction de diverses informations ou indices contextuels.

\subsection{Argumentativité}

En raison de ce qui précède, la pertinence discursive des énoncés d'expérience est en réalité essentiellement argumentative. A cet égard, ces énoncés sont passablement différents des énoncés narratifs. L'énoncé d'expérience sert en général à étayer, à justifier un autre énoncé, antérieur, ultérieur ou implicite. Cette fonction discursive est tout à fait évidente dans beaucoup des exemples donnés plus haut. Dans (13) (j'ai déjà eu un enfant...), l'énoncé d'expérience sert à justifier un savoir actuellement valide (je sais que ce moment est terrible où...); dans (15) ( $j$ 'ai souvent vu des fillettes très jeunes...), l'évocation de l'expérience consistant à avoir vu des fillettes qui avaient à l'égard des hommes une attitude typiquement féminine, est donnée comme une justification de l'affirmation suivant laquelle il y a une ressemblance entre fillettes et femmes adultes également du point de vue du comportement. L'exemple suivant est également caractéristique de ce fonctionnement argumentatif des énoncés d'expérience :

(21) Beaucoup d'artistes célèbres ont eu des manies bizarres aux heures du travail. Balzac s'en attribuait plus qu'il n'en avait réellement, et on lui en a prêté plus encore. Je l'ai surpris plus d'une fois, en plein jour, travaillant comme tout le monde, sans excitants, sans costume, sans aucun signe d'enfantement douloureux, riant dès l'abord, l'œil limpide et le teint fleuri. (G. Sand, Histoire de ma vie, Frantext)

Cherchant à étayer l'affirmation suivant laquelle les manies de Balzac étaient en partie de la mystification, G. Sand signale qu'elle l'a plusieurs fois surpris en plein jour, travaillant comme tout le monde, sans excitants, etc. Ce témoignage est apporté comme une justification du jugement énoncé antérieurement (Balzac s'en attribuait plus qu'il n'en avait réellement...).

Ce même fonctionnement argumentatif se rencontre avec plusieurs exemples de nos surcomposé-E. C'est en particulier le cas des items (9)-(11), répétés ci-après :

(9) Tous les Habitans sont Catholiques Romains. L'Evêque, souverain du Pays, a des revenus très-considérables. Il a eu mis dix mille hommes sur pied, en tems de guerre. (H. Descombes, Géographie universelle, T.1, Lausanne, 1790, p. 433)

(10) Les chaleurs de l'Eté n'y sont pas moins grandes que le froid de l'hyver; [...] Elles n'y sont, d'ailleurs, tempérées par aucun vent. On assure qu'elles ont eu mis des forêts en feu; \& que ces incendies ont, quelquefois, duré plusieurs mois, \&, même, plusieurs années, sans qu'on ait pû les éteindre. (ibid, p. 169)

(11) Ça serait un peu correct de ne pas mettre tout ce qui constitue l'alimentation bio dans le même panier. J'ai eu trouvé, dans un magasin bio, des steaks hachés sous barquette indiquant $12 \%$ de bio. Le reste est constitué de quoi ? (http ://www.tarot-numerologie.fr/)

Dans (9), l'assertion selon laquelle l'évêque a des revenus importants est étayée par le fait qu'il ait parfois mis dix mille hommes sur pied; dans (10), l'importance des chaleurs de l'été explique qu'elles ont parfois mis des forêts en feu ; dans (11), une consommatrice, cherchant à justifier l'assertion suivant laquelle 
tout ce qui est présenté comme bio ne doit pas être mis « dans le même panier», rapporte qu'il lui est arrivé de trouver dans un magasin bio des steaks hachés sous barquette indiquant $12 \%$ de bio. Ces exemples concordent tout à fait avec les observations rapportées par Jolivet (1986), qui note qu'avec le surcomposé régional (notre surcomposé-E), on peut rencontrer, outre des adverbes comme des fois, toujours, déjà, des adverbes argumentatifs comme pourtant ou quand même.

\subsection{Généralisation}

Le dernier point évoqué dans cette énumération est un peu différent, car il ne concerne pas la comparaison du passé composé et du surcomposé. Il s'agit du fait que les énoncés d'expérience ne se limitent pas en français à ces deux tiroirs. Le plus-que-parfait et le futur antérieur ont eux aussi des emplois bien attestés en énoncé d'expérience. Ce constat nous conforte dans l'idée que c'est bien, fondamentalement, la valeur de parfait dans ses diverses manifestations qui est concernée ici.

(22) J'étais bien fatiguée, nous voyagions par une chaleur étouffante, et mon premier mouvement fut de me jeter tout de mon long sur la natte en entrant dans la chambre qui nous était ouverte. Probablement nous avions déjà eu sur cette terre d'Espagne bouleversée par l'insurrection, des gîtes moins confortables, car ma mère s'écria : «à la bonne heure ! Voici des chambres très propres, et j'espère que nous pourrons dormir.» (G. Sand, Histoire de ma vie, Frantext)

(23) Quand je serai de nouveau loin de vous, cette nuit même, je prierai à vos intentions, en aveugle, et je n'aurai jamais prié de si bon cæur. (G. Bernanos, Sous le soleil de Satan, Frantext)

Les séquences en italiques de ces extraits sont très clairement des énoncés d'expérience, ne différant des cas déjà examinés que par le fait que, pour (22), la résultativité de l'énoncé est localisée dans le passé, et pour (23), dans le futur. $C f$. les transpositions avec le tour impersonnel :

(22a) Probablement il nous était déjà arrivé d'avoir sur cette terre d'Espagne [...] des gîtes moins confortables...

(23a) je prierai à vos intentions, en aveugle, et jamais il ne me sera arrivé de prier de si bon cœur.

On voit qu'en interprétation d'énoncé d'expérience, l'intervalle de validation du plus-que-parfait s'étend jusqu'à une limite terminale qui est elle-même localisée dans le passé ; celui du futur antérieur s'étend quant à lui jusqu'à une limite terminale localisée dans le futur. La limite initiale de l'intervalle de validation est, dans les deux cas, située dans le passé.

\section{Passé composé et passé surcomposé : points communs et différences}

Parmi les propriétés examinées dans la section précédente, deux semblent distinguer le passé composé en énoncé d'expérience et le surcomposé-E : leur rapport avec certains adverbiaux, et la définition de l'intervalle de validation. Examinons successivement ces deux problèmes. 


\subsection{Les adverbiaux}

Concernant les adverbiaux, nous nous livrerons à deux manipulations complémentaires : d'une part la substitution surcomposé-E $\rightarrow$ passé composé, d'autre part la suppression de l'adverbial accompagnant le passé composé.

\section{La substitution : surcomposé-E $\rightarrow$ passé composé}

Nous avons déjà noté en 3.1. que la substitution surcomposé-E $\rightarrow$ passé composé induisait parfois une ambiguïté. En réalité, on constate que cette substitution met fréquemment en cause la lecture expérientielle au profit d'une lecture prétéritale du passé composé, parfois même d'une lecture accomplie. Voici quelques exemples de surcomposés-E avec lesquels se produit ce phénomène.

(24) Nous faisons toujours des tranchées du côté de St Mihiel, nous avons eu travaillés jusqu'à très tard le soir (lettre de soldat, 1915. In Géa, 1995 : 76)

(25) Malory est plus susceptible et une petite tape non intentionnelle finit en mélodrame alors que dans ses périples de casse cou elle s'est eu fait bien plus mal. (http ://blog.aufeminin.com/)

(26) Sinon sur le site de l'ANPE il y a de temps en temps des offres d'emploi (j'en ai eu trouvé de très intéressantes mais je suis sans réponse) (http ://btsdietetique.forumactif.com/)

(27) [en réponse à un message dans lequel un participant au forum signale qu'il s'inquiète de la disparition de son chat]

Depuis quand il a disparu? J'ai eu flippé aussi parce qu'il est arrivé que mon chat disparaisse pendant au moins 4-5 jours, ça fout les boules [...] (http ://www.etno$\mathrm{ka} . \mathrm{fr} /$ forums/)

Dans (24), un passé composé pourrait fort bien être interprété comme un prétérit, même après l'énoncé Nous faisons toujours des tranchées du côté de St Mihiel - ou toujours à vraisemblablement une valeur continuative (= "encore maintenant ») et non pas une valeur de quantificateur temporel universel. En dépit de ce contexte, qui favorise peut-être une interprétation comme énoncé d'expérience, on ne peut pas exclure que ce passé composé hérite d'une localisation temporelle précise qui aurait été formulée antérieurement ; auquel cas il serait interprété comme un prétérit. Il est intéressant de noter ici qu'une façon simple de rétablir l'interprétation existentielle, et même de la forcer, consiste simplement à ajouter un adverbe quantifieur de fréquence, par exemple parfois : la formulation nous avons parfois travaillé jusqu'à très tard le soir ne peut être lue, elle, que comme énoncé d'expérience.

Il en va un peu de même dans (25) et sa transposition au passé composé. Ici, c'est le pluriel du syntagme prépositionnel dans ses périples de casse cou qui incite assez naturellement à une interprétation comme énoncé d'expérience : il induit en effet une lecture itérative de l'énoncé au passé composé (de fait, ce circonstanciel spécifie l'intervalle de validation). Mais une interprétation comme prétérit n'est pas complètement impossible ici aussi. A nouveau, la combinaison d'un passé composé et d'un quantifieur de fréquence forcerait une lecture existentielle (dans ses périples de casse cou elle s'est parfois fait bien plus mal).

Dans (26) l'effet de la transposition est plus spectaculaire. Au passé composé, cet exemple serait certainement interprété comme un accompli du présent, signifiant donc à peu près « $j$ 'en ai actuellement de très intéressantes ». Ici aussi un parfois rétablirait la valeur d'énoncé d'expérience. 
Le cas de (27) est intéressant pour une autre raison. Le surcomposé y est en effet suivi par un énoncé d'expérience explicite, utilisant la construction impersonnelle il est arrivé que. Pourtant, ici encore, un passé composé à la place du surcomposé entraînerait une équivoque. Le passé composé $j$ 'ai flippé pourrait être interprété comme un prétérit, si par exemple le scripteur venait lui-même d'évoquer une récente disparition de son chat; $j$ 'ai flippé aussi et il est arrivé que mon chat disparaisse pendant au moins 4-5 jours désigneraient alors deux situations de disparition différentes, localisées à des périodes différentes. Mais ce même passé composé pourrait également être interprété comme un énoncé d'expérience ; les deux énoncés $j$ 'ai flippé aussi et il est arrivé que mon chat disparaisse pendant au moins 4-5 jours concerneraient alors à la même situation de disparition (comme dans l'exemple original). Et ici, à nouveau, la présence d'un adverbial quantifieur de fréquence (par ex.j'ai parfois flippé aussi) sélectionnerait automatiquement la seconde de ces lectures.

Au total, on ne peut qu'être frappé, au terme de ces manipulations, par les deux phénomènes suivants :

- d'une part, la solidarité qu'il y a entre ces adverbiaux et la lecture expérientielle du passé composé ;

- d'autre part, la quasi-équivalence sémantique qu'il y a entre : (i) la formulation au passé surcomposé, (ii) sa transposition au passé composé accompagnée de parfois, et (iii) l'enchâssement dans le tour impersonnel au passé composé, sans adverbial. Soit, en reprenant les exemples (24) à (27) ci-dessus :

- nous avons eu travaillé jusqu'à très tard le soir

- nous avons parfois travaillé jusqu'à très tard le soir

- il nous est arrivé de travailler jusqu'à très tard le soir

- dans ses périples de casse cou, elle s'est eu fait bien plus mal

- dans ses périples de casse cou, elle s'est parfois fait bien plus mal

- dans ses périples de casse cou, il lui est arrivé de se faire bien plus mal

- j'en ai eu trouvé de très intéressantes

- j'en ai parfois trouvé de très intéressantes

- il m'est arrivé d'en trouver de très intéressantes

- j'ai eu flippé aussi

- j'ai parfois flippé aussi

- il m'est arrivé de flipper aussi

\section{La suppression de l'adverbial accompagnant le passé composé}

La dépendance, certes relative mais incontestable, que manifeste le passé composé vis-à-vis de ces adverbiaux quand il est interprété en énoncé d'expérience, est confirmée par la manipulation consistant simplement à supprimer l'adverbial quand celui-ci porte sur un passé composé. Le résultat est presque toujours le même : une ambiguïté apparaît dans l'interprétation de l'énoncé. Il y a donc bien un fait de collocation entre cet emploi du passé composé et la présence d'un quantifieur de fréquence. En voici encore un exemple.

(28) J'ai vu plus d'une fois le dimanche, à la musique, certains petits chevaux qui semblaient détachés de la frise du Parthénon. (E. About, La Grèce contemporaine, 1854, Frantext)

(28a) J'ai vu le dimanche, à la musique, certains petits chevaux qui semblaient détachés de la frise du Parthénon. 
Si on ne considère de (28) que l'extrait retenu ici, la suppression de plus d'une fois produit l'effet suivant : 1'expression le dimanche, interprétée dans (28) comme une localisation temporelle à caractère « habituel», devient dans (28a) une localisation anaphorique définie ( $c f$. "le dimanche de la semaine que je suis en train d'évoquer »). En ce cas, j'ai vu le dimanche désigne une occurrence unique et temporellement localisée de la situation, d'où une interprétation du passé composé comme un prétérit de type narratif. Il est intéressant de noter que dans l'énoncé d'expérience (28), le dimanche est, de fait, l'expression de l'intervalle de validation, et que cet intervalle est ici discontinu, en raison de la lecture « habituelle» de le dimanche.

Certains emplois du passé composé échappent toutefois complètement à cette dépendance. Il s'agit par exemple de formulations comme j'ai vu mieux, j'ai vu pire, $j$ 'ai été jeune, qui sont pratiquement des énoncés d'expérience figés. Dans de telles formulations, qu'il serait intéressant de répertorier, un adverbial quantifieur de fréquence (comme parfois) ou de passé non défini (comme jadis) serait pratiquement pléonastique.

On voit la conclusion vers laquelle convergent ces observations : tout se passe comme si une certaine information, celle-là même qu'apportent les adverbiaux considérés ici, était en quelque sorte déjà codée par le surcomposé-E, mais non par le passé composé. Dès lors, cette information, quand elle n'est pas apportée par d'autres éléments contextuels, est nécessaire au passé composé pour sélectionner l'interprétation existentielle, alors qu'elle est superflue avec le surcomposé ${ }^{(13)}$. L'hypothèse que nous ferons est donc que la variante du passé surcomposé examinée ici (le surcomposé-E) grammaticalise - ni plus ni moins - la valeur d'énoncé d'expérience. Cela signifie que pour les locuteurs qui pratiquent cette variante, la valeur d'énoncé d'expérience est une overt category au sens de Whorf(1956), autrement dit une catégorie qui possède un moyen d'expression propre. En revanche cette valeur n'est, pour le passé composé, qu'un «emploi ». C'est un premier point permettant d'affirmer qu'il n'y a pas synonymie entre le passé composé d'expérience et le surcomposé-E.

Cette équivalence au moins partielle entre la séquence « surcomposé-E » et la séquence «passé composé + adverbial »s'observe parfois à l'intérieur d'un même texte et à courte distance. C'était le cas dans l'exemple (10): On assure qu'elles ont eu mis des forêts en feu; \& que ces incendies ont, quelquefois, duré plusieurs mois, \&, même, plusieurs années... On observe un phénomène analogue, mais dans l'ordre inverse, dans les deux exemples suivants :

(29) Je n'ai jamais beaucoup aimé les honneurs. J'ai souvent vu qu'on donnait des médailles, je ne préciserai pas, à certaines personnes et $j$ 'ai eu pensé que si c'était à moi qu'on donnait ce type de médaille, j'en serais déshonoré si j'acceptais... (www.euskonews.com/)

(30) [il est question d'un incubateur pour œufs de reptiles] sa fiabilité laisse à désirer. J'ai maintes fois réglé le thermostat sur $31-32^{\circ} \mathrm{C}$ pour les Testudo et plusieurs œufs ont été cuits car la température a eu monté jusqu'à $38^{\circ} \mathrm{C}$ ! (http://la-passion-des-tortues.ifrance.com)

Ces exemples nous confortent dans l'idée que, en interprétation comme énoncé d'expérience, il y a une réelle dépendance du passé composé relativement aux ad-

(13) Frei (1929 : 80) avait lui-même déjà noté le caractère pléonastique d'adverbiaux comme des fois ou dans le temps, quand ils accompagnent la variante de surcomposé qui nous intéresse ici. 
verbiaux quantifieurs de fréquence, ce qui n'est pas le cas du surcomposé-E. En principe cette observation devrait être corroborée statistiquement par une fréquence plus grande des adverbiaux en question avec le passé composé qu'avec le surcomposé. Ce qui semble bien être le cas ${ }^{(14)}$. Néanmoins certaines considérations viennent quelque peu nuancer cette conséquence apparemment logique :

- D'abord, en plus de leur valeur de quantificateur existentiel, ces adverbiaux portent d'autres informations, de telle sorte qu'il serait erroné d'affirmer qu'ils sont, quand ils accompagnent un surcomposé, entièrement tautologiques : j'ai eu vu plusieurs fois... n'est pas synonyme de j'ai eu vu parfois..., j'ai déjà eu vu..., etc. La composante existentielle du sens de ces adverbiaux n'est qu'une composante parmi d'autres.

- Ensuite, l'interprétation expérientielle du passé composé peut être induite par d'autres éléments contextuels encore, qui n'ont pas nécessairement une expression explicite. On 1'a vu plus haut en 3.1., et on a signalé également le rôle que peut jouer ici la prosodie.

- Enfin, dans les questions, le caractère plus ou moins exceptionnel ou inhabituel du contenu de la question peut à lui seul influencer l'interprétation. Toutes choses égales, une question comme Avez-vous mangé du chocolat? a moins de probabilités d'être interprétée comme un énoncé d'expérience qu'une question comme : Avez-vous mangé de la viande de castor ?...

\subsection{L'intervalle de validation}

Rappelons qu'il s'agit de l'intervalle temporel à l'intérieur duquel la prédication d'existence exerce son effet, et donc d'un paramètre indissociable de celle-ci. La question que nous allons poser est celle de l'extension de cet intervalle.

Décrivant le parfait d'expérience anglais, i.e. l'un des emplois du present perfect, Comrie (1976:58) écrit qu'il signifie «that a given situation has held at least once during some time leading up to the present ». On retrouve dans cette définition ce qui a été dit plus haut au point 3.2. : " has held at least once during some time ». Mais il y a ici une spécification supplémentaire : "leading up to the present ". L'intervalle de validation du present perfect anglais employé comme parfait d'expérience n'est borné à droite que par le présent de la parole. Il en va de même pour les énoncés d'expérience au passé composé français. Cela signifie, de fait, que la situation dont il est dit qu'elle s'est produite une fois au moins, est susceptible de se reproduire jusqu'au moment de la parole ${ }^{(15)}$.

(14) Il nous est difficile d'en dire davantage ici, pour la raison suivante : une façon commode de constituer un corpus de passés composés à valeur d'expérience consiste justement à les rechercher systématiquement en collocation avec un adverbial comme parfois, souvent, rarement, etc. Ce que nous avons fait, évidemment. Mais un corpus constitué ainsi est par définition biaisé si on veut quantifier cette collocation. Ce qui interdit ensuite toute comparaison avec le surcomposé-E. Par ailleurs, concernant le surcomposé, Jolivet (1986:112) observe le fait suivant, particulièrement intéressant mais difficile à interpréter : tandis qu'avec les surcomposés-E, les insertions d'adverbes se font généralement immédiatement avant eu (type : on a souvent eu vu...), ces mêmes insertions se font, avec l'autre surcomposé, entre l'auxiliation et le participe passé du verbe lexical (type : quand j'ai eu bien regardé...).

(15) Cette définition vaut pour les parfaits présents. Pour rendre compte des énoncés d'expérience formulés au plus-que-parfait ou au futur antérieur, dont nous avons vu des exemples dans la section 3.8, il faudrait généraliser la définition de Comrie en modifiant la dernière clause comme suit : «...durant une période s'étendant jusqu'à une certaine limite ». Cette 
Qu'en est-il de l'intervalle de validation du surcomposé-E ? Commençons par une observation générale.

Notre corpus montre que le surcomposé-E apparaît fréquemment dans des routines contrastives, où l'existence d'une situation passée est comparée avec ce qui peut être observé dans le présent. Quand elle a lieu, cette mise en contraste vise en général, implicitement ou explicitement, à faire savoir que la situation, attestée dans le passé, n'existe plus dans le présent (ou l'inverse). Exemples :

(31) Cette mine a eu produit jusqu'à 24 mille marcs d'argent fin, dans une année ; mais elle a beaucoup diminué. (H. Descombes, Géographie universelle, Lausanne, T.1, 1790, p. 188. Un marc est une ancienne unité de mesure du poids)

(32) Enfin, en ce qui concerne mes vacances, $j$ 'en ai eu passé de meilleures, n'étant pas parti cette année. (http ://forums.france3.fr/france3/france $3 \mathrm{jt} /$ )

(33) Je rentre de la chasse aux œufs avec mon fiston et le chien. Après $2 \mathrm{~h} 30$ de marche dans le village, on rentre crevés mais heureux. On a réalisé un beau score : 10 œufs. Il faut savoir qu'on est eu rentré avec un seul œuf... (http ://forum.aufeminin.com/forum/)

(34) cette année la rencontre a lieu à Lausanne, mais ça s'est eu fait à Paris, ça s'est eu fait à Lyon, ça s'est eu fait à Montréal (locuteur lausannois, 2008)

Dans (31), il y a comparaison entre la production d'une mine à une certaine époque et sa production actuelle; dans (32), entre les vacances dans le passé et les vacances de cette année; dans (33), entre certaines récoltes d'œufs faites dans le passé et la récolte qui vient d'être faite ; dans (34), entre la ville où une certaine rencontre a eu lieu dans le passé et la ville où elle a lieu cette année. L'exemple (25) présentait lui aussi cette routine contrastive.

Ce phénomène est difficile à quantifier, car la comparaison peut être implicite et donc difficilement identifiable. Toutefois, selon nos calculs, nous estimons que près de la moitié des exemples de surcomposés existentiels de notre corpus figurent dans un contexte de ce type (estimations faite sur un corpus de quelque cent items).

Autre observation : la mise en contraste entre passé et présent s'effectue souvent sur un paramètre quantifiable, du moins gradable. C'est clairement le cas dans (31)-(33), où il est question respectivement : de la quantité d'argent produite par une mine ; de l'évaluation de la qualité des vacances ; du nombre d'œufs récoltés. L'exemple (34) échappe à cette logique mais n'en comporte pas moins une comparaison mettant en jeu passé et présent. La confrontation du passé et du présent relativement à un paramètre quantifiable est également une caractéristique des exemples suivants :

(35) Je décortique les secondes, et plus elles passent, plus je me dis que je n'ai jamais vu passer le temps aussi vite! C'est vrai, alors quej'ai eu trouvéles minutes longues, voilà que maintenant les heures sont courtes !

(http ://ruptures.bleublog.lematin.ch/)

(36) - L'album est bon mais ne comprend que neuf titres. Pourquoi si peu ?

- Je pense que c'est assez. On a eu fait de longs albums et ça peut être ennuyeux d'écouter un album pendant plus d'une heure. On voulait faire comme beaucoup de groupes des années 80 et livrer quarante minutes de bande. Je pense que cet album est parfait ainsi, dans sa durée. (http :/www.zicazic.com/zicazine/)

limite est située dans le passé dans le cas du plus-que-parfait, et dans le futur dans le cas du futur antérieur, comme nous l'avons signalé plus haut en 3.8. 
Sans avoir mené sur ce point une investigation systématique, il nous semble pouvoir affirmer que cette co-présence du contraste passé/présent et d'un paramètre quantifiable est un trait caractéristique de l'environnement sémantique du surcomposé-E, même si on ne la rencontre pas dans tous les cas.

Quelles conclusions peut-on tirer de ces observations, concernant l'intervalle de validation? Selon nous, le fait suivant : si les énoncés au surcomposé-E sont si fréquemment associés à l'expression d'une relation de contradiction entre passé et présent, c'est que leur intervalle de validation est, contrairement à celui du passé composé, entièrement situé dans le passé. Autrement dit, la limite droite de cet intervalle (son terminus ad quem) est elle-même localisée dans le passé. Si cette analyse est correcte, elle implique que la situation désignée par ces formes verbales n'est pas présentée comme susceptible de se reproduire jusqu'au moment de l'énonciation (contrairement au passé composé).

L'exemple (35) est particulièrement intéressant de ce point de vue. Il comporte successivement un passé composé en énoncé d'expérience (je n'ai jamais vu passer le temps aussi vite) et un surcomposé-E (alors que j'ai eu trouvé les minutes longues). Mais, tandis que l'intervalle de validation du passé composé s'étend jusqu'au présent de l'énonciation ("dans le passé et jusqu'à aujourd'hui je n'ai jamais vu passer le temps aussi vite »), l'intervalle de validation du surcomposé-E s'étend jusqu'à une limite située elle-même dans le passé ("dans le passé et jusqu'à une date située dans le passé, il m'est arrivé de trouver les minutes longues »). La localisation dans le passé du terminus ad quem de l'intervalle de validation paraît bien être ce qui fait la spécificité du surcomposé-E relativement à celle du passé composé.

Ce positionnement dans le passé de l'intégralité de l'intervalle de validation est parfois explicitement signalé :

(37) j'ai eu rencontré ce pbm chez quelques clients fut un temps... (http ://www.hyjoo.com/sujet-29288.html)

(38) - Vertige nausée, ça ressemble à l'oreille interne.

- Oui c'est effectivement probablement l'oreille interne mais le problème est de trouver pourquoi ? En effet elle a eu fait des vertiges de positionnement par le passé [...]. (http ://www.plongeur.com/forums/)

\section{Bilan et discussion}

On peut résumer ce qui précède de la façon suivante. Nous sommes partis de l'observation selon laquelle il y a une forte convergence sémantique (une synonymie) entre certains emplois du passé composé et le passé surcomposé dans la variante couramment appelée « régionale » (nommée ici « surcomposé-E »). Cette convergence s'explique par le fait que tous deux sont des parfaits d'expérience au sens de Comrie (1976), que tous deux construisent ce que nous avons appelé des " énoncés d'expérience ».

Cependant, deux caractéristiques au moins amènent à distinguer ces deux formes : la première relève de la grammaire, la seconde de la sémantique.

En premier lieu, nous avons apporté un certain nombre d'arguments montrant que la relation que le passé composé et le surcomposé-E entretiennent avec les énoncés d'expérience est radicalement différente. Tandis que, pour le passé composé, il ne s'agit que d'une valeur parmi d'autres, nécessitant la collaboration de données contextuelles particulières pour s'exprimer, pour le surcomposé-E il s'a- 
git au contraire d'une valeur en quelque sorte intrinsèque. En un mot, le surcomposé-E grammaticalise la valeur d'énoncé d'expérience. Il en découle que, pour les sujets parlants qui « possèdent » ce tiroir, il appartient à l'ensemble des moyens grammaticaux permettant de produire un énoncé d'expérience.

En second lieu, nous avons formulé l'hypothèse, fondée sur certaines observations, suivant laquelle passé composé (en énoncé d'expérience) et surcomposé-E diffèrent par leur intervalle de validation. Dans le cas du passé composé, cet intervalle s'étend jusqu'au moment de l'énonciation, qui en constitue le terminus ad quem par défaut. Cette propriété implique, comme certains auteurs 1'ont relevé, que la situation désignée ainsi est présentée comme ressortissant à un type et qu'elle peut encore se reproduire. En revanche, dans le cas du surcomposé-E, le terminus ad quem de l'intervalle de validation est localisé par défaut dans le passé, c'est-à-dire avant le moment de l'énonciation. Cette différence explique vraisemblablement pourquoi ce tiroir est si souvent utilisé dans des routines discursives où s'exprime un contraste temporel entre le passé et le présent, cette expression s'accompagnant généralement du sous-entendu suivant lequel ce qui est affirmé du passé ne saurait se répéter dans le présent.

Cette présentation pourrait donner l'impression que la situation du surcomposé-E, au plan linguistique, est relativement claire. Mais en réalité plusieurs incertitudes demeurent. Celle dont nous voudrions dire quelques mots est la suivante.

En réalité, il n'est pas certain du tout que le surcomposé-E soit toujours employé exactement de la façon décrite plus haut, spécialement en ce qui concerne l'intervalle de validation. Cette incertitude est liée au statut « dialectal» de cette variante de surcomposé. Il nous semble que deux situations peuvent être envisagées sur un plan purement théorique.

- Soit, dans les régions où il est pratiqué, le surcomposé-E constitue un instrument grammatical complémentaire pour produire les énoncés existentiels, à côté du passé composé et d'autres moyens encore, avec la différence décrite plus haut concernant la définition de l'intervalle de validation. Cela signifierait que pour les locuteurs qui le pratiquent, le système des tiroirs verbaux grammaticalise une distinction qui est exprimée, pour les locuteurs qui ne le pratiquent pas, par d'autres moyens que la conjugaison (par exemple par des adverbiaux).

- Soit, dans les régions où il est pratiqué, il tend à supplanter, en énoncé d'expérience, le passé composé - voire le supplante complètement. En ce cas, il n'est pas du tout certain que les sujets parlants qui pratiquent le surcomposé-E fassent la différence que nous avons décrite concernant l'intervalle de validation. En terme de système, il serait au contraire plus logique qu'ils ne la fassent pas. La distinction des deux types d'intervalles de validation (l'un s'étendant jusqu'au présent, l'autre étant entièrement localisé dans le passé) s'exprimerait alors par le moyen de la complémentation adverbiale et ne dépendrait pas d'un choix de tiroir.

Cela dit, cette distinction est toute théorique. La plupart des locuteurs sont en contact avec une multitude de micro-variantes sociolectales et dialectales, qui passent d'ailleurs la plupart du temps complètement inaperçues. De telle sorte que quand il y a ce type de variation, la situation ne peut pas être décrite en des termes aussi tranchés que ne le fait l'alternative exposée ci-dessus. En réalité, seules des études sur le terrain - et des études beaucoup plus détaillées que celles mises en œuvre jusqu'ici - permettraient d'en savoir davantage sur cette question.

Concernant la synonymie, on entrevoit à quel point le problème que nous avons examiné est complexe. Si l'on s'en tient simplement aux deux situations théori- 
ques esquissées plus haut, alors dans les deux cas il n'y a qu'une synonymie partielle.

Dans la première situation, le surcomposé-E se distingue du passé composé sur trois paramètres : (i) il encode la valeur d'énoncé d'expérience, celle-ci étant ainsi une overt category, alors qu'elle n'est qu'un « emploi » pour le passé composé ; (ii) son intervalle de validation par défaut est intégralement localisé dans le passé, ce qui n'est pas le cas du passé composé ; (iii) il n'est pratiqué que sur un territoire relativement limité, avec tout ce que cela peut entraîner au plan sociolinguistique.

Il en va tout autrement dans la seconde situation. En admettant que pour certains locuteurs, le surcomposé-E supplante complètement le passé composé pour les énoncés d'expérience (hypothèse évidemment purement théorique), il y aurait alors une vraie synonymie entre les deux tiroirs. Mais ce constat doit immédiatement être nuancé sur deux points : d'une part, cette synonymie n'apparaîtrait que dans la comparaison de deux « systèmes » différents ; d'autre part, elle concernerait une signification qui est une overt category dans l'un des systèmes (le dialecte), et une covert category dans l'autre (le système réputé standard). Comme on le voit, le point délicat ici est évidemment celui de la comparaison entre une forme qui grammaticalise une valeur, et une forme dont on ne considère qu'un emploi parmi d'autres.

\section{Références}

A POTHÉlOZ, D. (à par.) : « Le passé surcomposé et la valeur de parfait existentiel », Journal of French Language Studies (accepté).

A pothÉloz, D., NowAKOWSKA, M. (2009) : « Note sur la résultativité et la valeur de parfait : leur expression en polonais », Cahiers Chronos, 21 (sous presse).

A YRES-BENNETT, W., CARRUTHERS, J. (1992) : " "Une regrettable et fort disgracieuse faute de français" ? : The description and analysis of the French surcomposés from 1530 to the present day », Transactions of the Philological Society, 90/2, 219-257.

BArceló, G.J., Bres, J. (2006) : Les temps de l'indicatif en français, Paris : Ophrys.

BENVENISTE, E. (1959) : «Les relations de temps dans le verbe français », Bulletin de la Société de Linguistique, LIV, fasc. 1. Repris dans Problèmes de linguistique générale, 1. Paris : Gallimard, 1966, 237-250.

BÜRGI, A. (2007) : «Analyse de l'émergence et du sens du surcomposé », in: P. Larrivée (éd.), Variation et stabilité du français. Des notions aux opérations, Louvain : Peeters, pp. 33-50.

CARRUTHERS, J. (1994) : «The passé surcomposé régional : towards a definition of its function in contemporary spoken French », Journal of French Language Studies, 4, 171-190.

— (1998) : "Surcomposé "général" et surcomposé "régional": deux formes distinctes ? », in: G. Ruffino (ed.), Atti del XXI Congresso Internazionale di Linguistica e Filologia Romanza, Vol. 2, Tübingen : Max Niemeyer, pp. 143154.

- (1999) : "A problem in sociolinguistic methodology : investigating a rare syntactic form », Journal of French Language Studies, 9, 1-24. 
ClÉDAT, L. (1926) : «Les tens composés et surcomposés », Revue de philologie française et de littérature, 38, 33-47.

COMRIE, B. (1976) : Aspect. An introduction to the study of verbal aspect and related problems, Cambridge : Cambridge University Press.

CONFAIS, J.-P. (1995) : Temps, mode, aspect. Les approches des morphèmes verbaux et leurs problèmes à l'exemple du français et de l'allemand, Toulouse : Presses Universitaires du Mirail.

CORnU, M. (1953) : Les formes surcomposées en français, Berne : A. Francke.

D AHL, Ö. (1985) : Tense and aspect systems, Oxford : Blackwell.

DAUZAT, A. (1954) : «A propos des temps surcomposés : surcomposé provençal et surcomposé français », Le français moderne, 22, 259-262.

DesClÉS, J.-P., GUENTCHÉVA, Z. (2003) : « Comment déterminer les significations du passé composé par une exploration contextuelle », Langue française, 138, 48-60.

ENGEL, D.M. (1996) : « Le passé du passé », Word, 47/1, 41-62.

Foulet, L. (1925) : « Le développement des temps surcomposés », Romania, 51, 203-252.

FREI, H. (1929) : La grammaire des fautes, Bellegarde : Société anonyme des arts graphiques de France.

GÉA, J.-M. (1995) : «Entre norme et usage : quelques formes surcomposées dans les lettres de deux soldats méridionaux de la Grande Guerre », Langage et société, 71, 65-85.

Gosselin, L. (2005) : Temporalité et modalité, Bruxelles : De Boeck \& Larcier.

Gougenheim, G. (1939) : Système grammatical de la langue française, Paris : d'Artrey.

GuENTCHÉVA, Z. (1990) : Temps et aspect : l'exemple du bulgare contemporain, Paris : Editions du CNRS.

HiLL, J.K. (1984) : " "A la recherche de temps perdus": The double compound forms of the verb in present-day French », Word, 35/1, 89-112.

Jespersen, O. (1924) : The Philosophy of Grammar, London : George Allen \& Unwin Ldt. Trad. franç. La philosophie de la grammaire, Paris : Les Editions de Minuit, 1971.

JOLIVET, R. (1984) : «L'acceptabilité des formes verbales surcomposées », Le français moderne, 52, 159-176.

— (1986) : "Le passé surcomposé : emploi "général” et emploi "régional". Examen des insertions dans le syntagme verbal surcomposé », in: Mélanges d'onomastique, linguistique et philologie offerts à M. Raymond Sindou, tome II : Linguistique et philologie, Milliau : Presses de la Société des imprimeurs Maury, pp. 109-116.

KAROLAK, S. (2007) : Składnia francuska o podstawach semantycznych, tom 1, Kraków : Collegium Columbinum.

KLEIBER, G. (2001) : "Indéfinis : lecture existentielle et lecture partitive », in : G. Kleiber, B. Laca et L. Tasmowski (sous la dir. de), Typologie des groupes nominaux, Rennes : Presses Universitaires de Rennes, pp. 47-97.

LAMBRECHT, K. (1994): Information Structure and Sentence Form. Topic, focus, and the mental representations of discourse referents, Cambridge : Cambridge University Press.

LEECH, G.N. (1971) : Meaning and the English verb, London : Longman. 
MCCAWLEY, J.D. (1971) : «Tense and time reference in English », in : C.J. Fillmore, D.T. Langendoen (eds), Studies in linguistic semantics, New York : Holt, Rinehart and Winston, 96-113.

MARTINET, A., sous la dir. de (1979) : Grammaire fonctionnelle du français, Paris : Crédif-Didier.

MosegaArd Hansen, M.-B. (2000) : «La polysémie de l'adverbe déjà », in : H.L. Andersen, A.B. Hansen (éds), Le français parlé : corpus et résultats, Cøbenhavn : Museum Tusculanum Press, 157-177.

Nilsson-Ehle, H. (1953) : «Remarques sur les formes surcomposées en français », Studia Neophilologica, 26 (1-3), 157-167.

REICHENBACH, H. (1947) : Elements of symbolic logic, London \& New York : Macmillan.

RITZ, M.-E. (2002) : "The semantics of the passé composé in contemporary French : towards an unified representation », Cahiers Chronos, 9, 31-50.

SCHADEN, G. (2007) : La sémantique du parfait. Etude des «temps composés » dans un choix de langues germaniques et romanes, Thèse de doctorat, Université Paris 8.

SQuartini, M. (1998) : Verbal Periphrases in Romance. Aspect, Actionality, and Grammaticalization, Berlin, New York : W. de Gruyter.

STEFANINI, J. (1954) : «La tradition grammaticale française et les temps surcomposés ", Annales de la Faculté des Lettres d'Aix, 28, 67-108. Réédité dans : Linguistique et langue française. Textes de J. Stefanini réunis par P. Bonnefois, Paris : Editions du C.N.R.S., 1992, pp. 37-74.

STHioul, B. (2006) : «Le(s) passé(s) surcomposé(s) : temps, aspect, subjectivité », Travaux neuchâtelois de linguistique, 45, 115-132.

TESNiÈre, L. (1939) : "Théorie structurale des temps composés », in: Mélanges de linguistique offerts à Charles Bally, Genève : Georg, pp. 153-183.

VAn DE VELDE, D. (2006) : Grammaire des événements, Villeneuve d'Ascq : Presses Universitaires du Septentrion.

VET, C. (1992) : «Le passé composé : contextes d'emploi et interprétation », Cahiers de Praxématique, 19, 37-59.

WALTER, H. (1981) : «Le surcomposé dans les usages actuels du français », in : Actants, voix et aspects verbaux, Angers : Presses de l'Université d'Angers, pp. 24-44.

WAUGH, L.R. (1987) : « Marking time with the passé composé : toward a theory of the perfect », Linguisticae Investigationes, 11, $\mathrm{n}^{\circ}$ 1, 1-47.

WEINRICH, H. (1989) : Grammaire textuelle du français, Paris, Didier.

WhORF, B.L. (1956) : Language, thought and reality, Cambridge (Mass.) : The M.I.T. Press.

WiLMET, M. (1997) : Grammaire critique du français, Louvain : Duculot. 